\title{
Histological Detection of mmcp-4 Expressing Cells by In Situ RT-PCR Method
}

\section{Tetsuya Tanaka ${ }^{1}$, Satoshi Asano ${ }^{2}$, Tomomi Niibayashi ${ }^{1}$, Haruki Usuda ${ }^{1}$, Takayuki Okamoto ${ }^{1}$, Koichiro Wada ${ }^{1}$}

\section{${ }^{I}$ Department of Pharmacology, Shimane University Faculty of Medicine, Japan, ${ }^{2}$ Department of Pharmaceutical Sciences, International University of Health and Welfare, Japan}

Mature mast cells (MCs) are not in the circulating blood. After reaching the target tissues, circulating MC precursors differentiate, maturate, and start to express many enzymes, histamine, chemical mediators and cytokines, and so on. Such many products are accumulated in their intracellular granules. Getting the activation signals, MCs release the contents in their granules to microenvironment in where they exist. Subsequently, some contents, mainly histamine, trigger immunological reaction, such as the allergic reaction, and some contents work for reconstitution of damaged tissues and for other biological functions.

It is known that mature MCs are highly heterogenic cells. In other words, one MC population expresses a specific gene set and other MC populations express other sets of genes. Commonly, MCs has been classified into two subtypes, which are connective-tissue-type MCs (CTMCs) expressing mmcp-4 and 5 specifically, and mucosal-type MCs (MTMCs) expressing mmcp-1 and 2 specifically.

For clarifying the function of each gene product in MCs, it is very important to define the genes expressed in each MC population on the histological samples. Although immunohistological or in situ hybridization method was used for such purpose, it seems that these methods have not enough sensitivity for all cases. For the detection of the mRNA on the tissue sample by in situ hybridization, at least 10 copies of target mRNAs per cells are generally required. On the other hand, it is reported that only one copy of mRNA is able to visualize by in situ RT-PCR method.

In this study, we amplified the mouse mast cell protease (mmcp) - 4 mRNAs by in situ RT-PCR method and successfully visualized the signals of amplicons. It was found that the cells expressing mmcp-4 mRNA were connective tissue type mast cells but not mucosal type mast cells, in mouse tongue and intestine sections. This is the first report that the cells expressing the mast cell-specific gene were detected on histological specimens by in situ RT-PCR method. And it is suggested that this method will be a powerful tool for clarifying the relationships between pathological and physiological changes in diseases and many genes expressed in mast cells. 